\title{
Analytic Formulas for Full Steiner Trees
}

\author{
R. S. Booth \\ The School of Mathematical Sciences, The Flinders University of South Australia, \\ Bedford Park, S.A. 5042, Australia
}

\begin{abstract}
It was conjectured by Gilbert and Pollak [6] that, for any finite set of points in the Euclidean plane, the ratio of the length of a Steiner minimal tree to the length of a minimal spanning tree is at least $\sqrt{3} / 2$. To date, this has been proved only for at most five points. In this paper, some analytic formulas for the length of full Steiner trees are considered. These provide an alternative proof of the conjecture for quadrilaterals, and the foundation for a possible approach for more complicated polygons.
\end{abstract}

\section{Introduction}

Let $P$ be a set of $n$ points in the Euclidean plane. Denote by $L_{\mathrm{m}}(P)$ the length of the minimal spanning tree of $P$, and by $L_{\mathrm{s}}(P)$ the length of the Steiner minimal tree of $P$. It was conjectured by Gilbert and Pollak [6] that

$$
L_{\mathrm{s}}(P) \geq(\sqrt{3} / 2) L_{\mathrm{m}}(P)
$$

for all $P$. At present, this has only been verified for $n \leq 5$. The case $n=3$ is easy. Pollak [9] provided the first proof for $n=4$, by considering all possible patterns for minimal trees. In subsequent papers, Du et al., provided a very much simpler proof for $n=4$ [4] and, later, proved the case $n=5$ [5]. Generalizations for $n \geq 6$ do not seem to be available yet.

The example of an equilateral triangle shows that $\sqrt{3} / 2$ cannot be replaced by a larger number, while in the opposite direction, it has been proved by Chung and Graham [1] with computer-edited computations, that $L_{\mathrm{s}}(P) \geq 0.824 L_{\mathrm{m}}(P)$ for all networks in the plane. It is also well known [6] that in order to prove the Gilbert-Pollak conjecture, only full Steiner trees need be considered.

In the present paper we derive some analytic formulas for $L_{\mathrm{s}}(P)$. If $P$ consists of four points these provide an alternative proof of the results of Du et al. 


\section{Preliminary Results}

We recall that a given set of vertices may have many full Steiner trees. Here, we wish to consider just one "topology" or full Steiner configuration, associated with the given set of vertices. Thus we assume that we are given a set of $m+1$ vertices which we label $A_{0}, A_{1}, \ldots, A_{m}$, and $m-1$ Steiner points $S_{1}, S_{2}, \ldots, S_{m-1}$, always two less in number than the given vertices together. We may assume [6] that each vertex $A_{i}$ is joined by a line segment to exactly one Steiner point, and each Steiner point is an endpoint of three segments, meeting mutually at $120^{\circ}$.

It is well known [6], [7] that there exist at least two distinct pairs of vertices $A_{i}$, where each of the pair is joined to the same Steiner point. Label the two vertices from one pair as $P_{0}, P_{1}$, and the corresponding Steiner point $S_{1}$ in such a way that the path $P_{0} S_{1} P_{1}$ changes angle by $60^{\circ}$ clockwise at $S_{1}$. Now the remaining vertices may be relabeled uniquely and unambiguously as $P_{2}, P_{3}, \ldots, P_{m}$, by requiring that the unique path from the vertex $P_{k}$ via the Steiner points to the vertex $P_{k+1}$, always changes angle by $60^{\circ}$ clockwise at each Steiner point. For convenience, we denote $P_{m+1}=P_{0}$. Now label the remaining Steiner points as $S_{2}, \ldots, S_{m-1}$, any of the possible permutations may be selected here. For illustrations, see Figs. 1 and 2. For each integer $k, 0 \leq k \leq m$, the path as described from $P_{k}$ to $P_{k+1}$ is denoted by $\hat{P}_{k}$. Notice that exactly three of these clockwise paths, one for each pair of segments meeting it, pass through each Steiner point.

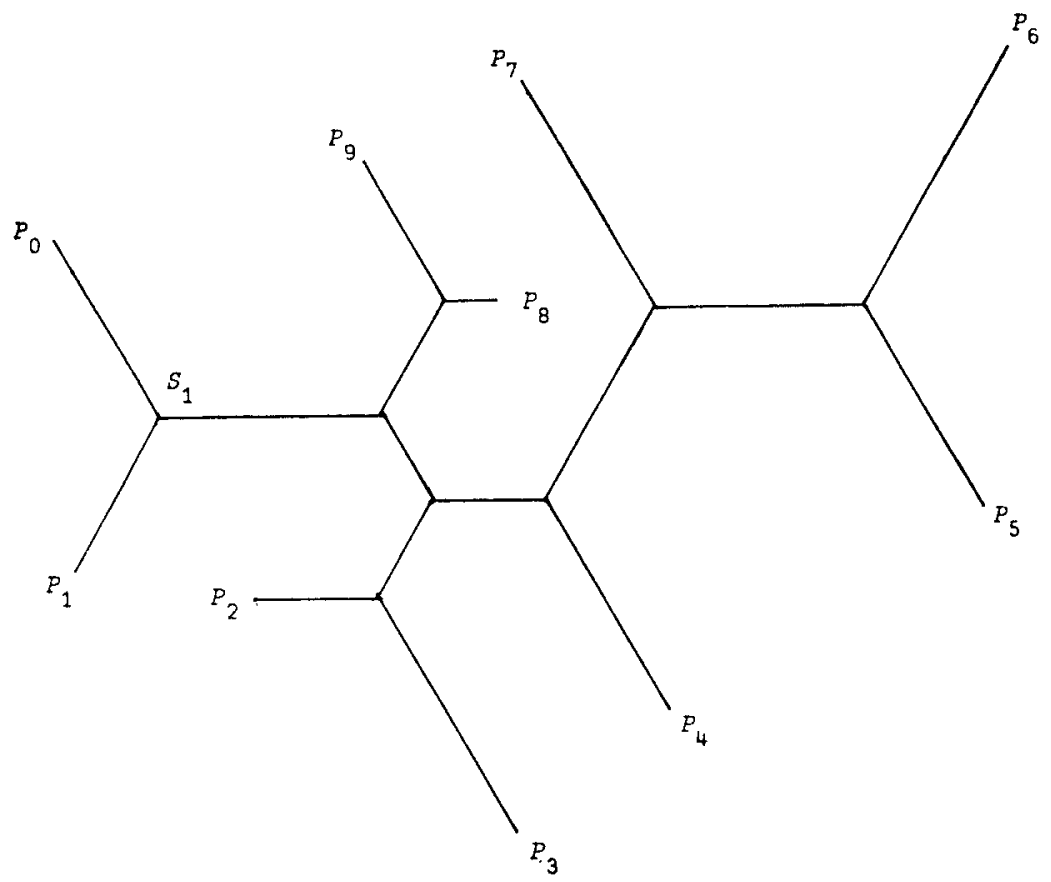

Fig. 1 


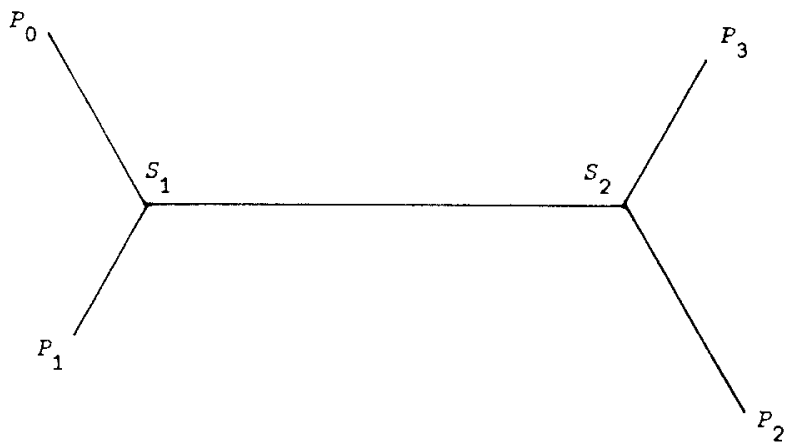

Fig. 2

Now observe that if $m \geq 3$, then the paths $\hat{P}_{1}$ and $\hat{P}_{m}$ meet in a common segment $S_{1} S_{2}$ joining two Steiner points. A unique path $\hat{P}_{q}, q \neq 1, q \neq m$, unique since three paths pass through $S_{2}$, and two of these are $\hat{P}_{1}$ and $\hat{P}_{m}$, also passes through $S_{2}$. Clearly, $1<q<m$.

If $q \geq 3$, then the paths $\hat{P}_{1}$ and $\hat{P}_{q}$ also share a common segment which has $S_{2}$ at one end, and another Steiner point $S_{3}$ at the other. There is then a unique third path through $S_{3}, \hat{P}_{q^{\prime}}$, defining $q^{\prime}$ such that $1<q^{\prime}<q$. Similarly, if $m-q \geq 2$, there is a corresponding path $\hat{P}_{q^{\prime \prime}}$ with $q<q^{\prime \prime}<m$.

The procedure can clearly be repeated until all paths, $\hat{P}_{k}, 1 \leq k \leq m$, are accounted for. The procedure, in fact, describes how, given certain integers $s, t$ with $t-s \geq 2$, and $1 \leq s<t \leq m$, an integer $q$ is defined, $s<q<t$ so that $\hat{P}_{s}, \hat{P}_{q}$,

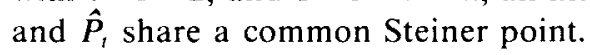

Define distances by

$$
\begin{array}{ll}
a_{k}=\left|P_{k} P_{k+1}\right| & \text { if } \quad k=0,1,2, \ldots, m, \\
a_{j k}=\left|P, P_{k}\right| & \text { if } \quad 0 \leq j, \quad k \leq m+1 .
\end{array}
$$

For angles, let $\overrightarrow{P_{0} P_{1}}$ be the vector defining the direction of the positive $x$-axis in the usual sense. $S_{1}$ is then in the upper half-plane. With this coordinate system, define $\beta_{k}$ to be the angle of the vector $\overrightarrow{P_{k} P_{k+1}}$, measured anticlockwise. Indeed, by regarding the $x$-axis as the real axis of the complex plane, then, via a translation, $\beta_{k}$ is such that

$$
\overrightarrow{P_{k} P_{k+1}}=a_{k} e^{i \beta_{k}}
$$

More generally, define angles $\beta_{j k}$ by $0 \leq \beta_{j k}<2 \pi$, and

$$
\overrightarrow{P_{j} P_{k}}=a_{j k} e^{i \beta_{1 k}}
$$

for

$$
0 \leq j, k \leq m+1 \text {. }
$$


Define also the angle $\lambda, 0<\lambda<\pi$, by

$$
\overline{P_{1} S_{1}}=\left|P_{1} S_{1}\right| e^{i \lambda}
$$

Finally, put $\omega=e^{i \pi / 3}$, and define the sequence $\left\{y_{q}\right\}, 1 \leq q \leq m$, inductively by:

(i) $y_{1}=1, y_{m}=\omega^{-1}$.

(ii) Given $y_{s}$ and $y_{t}, 1 \leq s<t \leq m$, then

$$
\omega^{-1} y_{s}+\omega y_{t}=y_{q}
$$

where $q$ is the integer, previously described, so that $s<q<t$ and $\hat{P}_{s}, \hat{P}_{t}$, $\hat{P}_{q}$ share a common Steiner point.

With the above notation, we can now state

Theorem 1. The length $z$ of the Steiner tree is given by the formula

$$
z e^{i \lambda}=\sum_{k=1}^{m} a_{k} y_{k} e^{i \beta_{k}}
$$

Proof. The proof is by induction on $m$. Assume that $m=2$. The configuration is then a triangle for which $y_{1}=1$ and $y_{2}=\omega^{-1}$. Draw the equilateral triangle $P_{0} P_{1} Q$, with the vertex $Q$ exterior to the given triangle $P_{0} P_{1} P_{2}$ (Fig. 3). By [7],

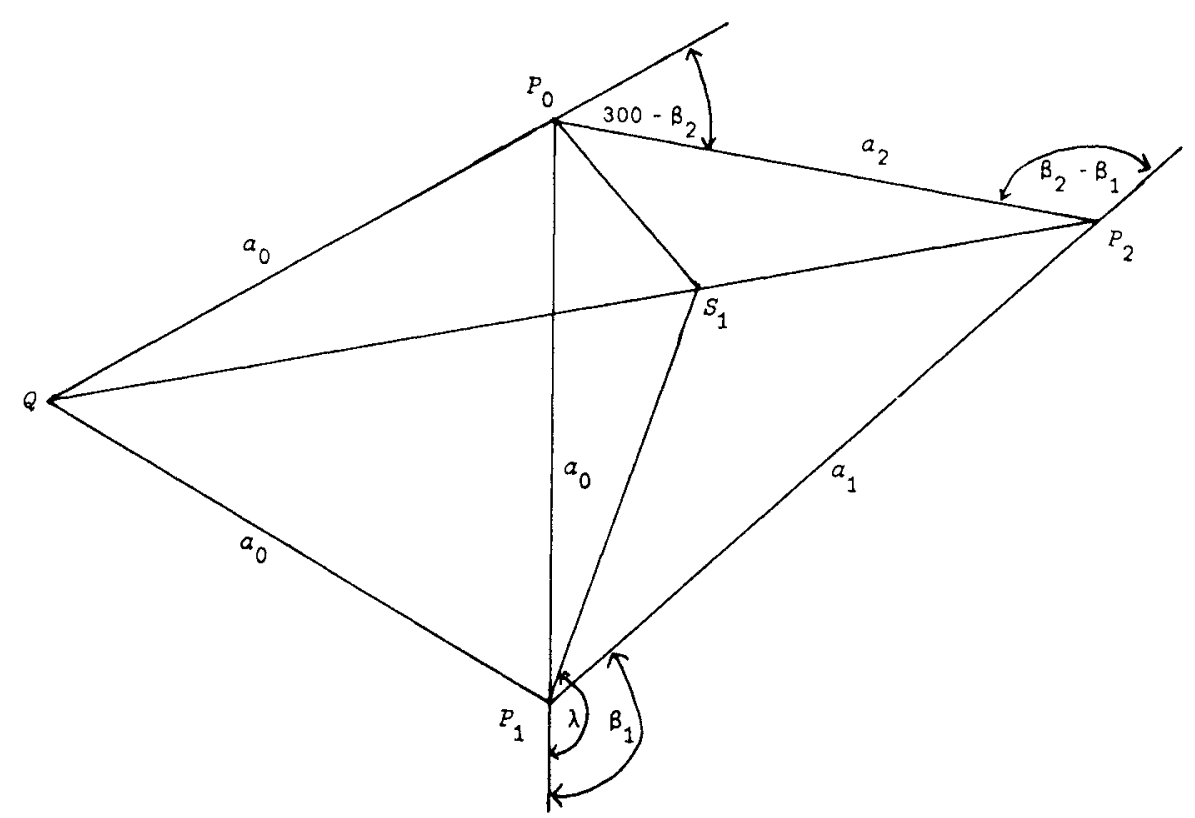

Fig. 3 
the points $P_{0}, S_{1}, P_{1}, Q$ are cocircular, hence

$$
\begin{aligned}
\angle P_{0} Q P_{2} & =\angle P_{0} Q S_{1} \\
& =\angle P_{0} P_{1} S_{1} \\
& =\pi-\lambda .
\end{aligned}
$$

Moreover [6], $z=\left|Q P_{2}\right|$. With a little elementary trigonometry,

$$
z \cos (\pi-\lambda)=a_{0}+a_{2} \cos \left(5 \pi / 3-\beta_{2}\right)
$$

and

$$
z \sin (\pi-\lambda)=a_{2} \sin \left(5 \pi / 3-\beta_{2}\right)
$$

so that

$$
z e^{i(\pi-\lambda)}=a_{0}+a_{2} e^{i\left(5 \pi / 3-\beta_{2}\right)} .
$$

But, by considering vector addition, we also have

$$
\begin{gathered}
a_{0}+a_{1} e^{i \beta_{1}}+a_{2} e^{i \beta_{2}}=0, \\
a_{0}+a_{1} e^{-i \beta_{1}}+a_{2} e^{-i \beta_{2}}=0 .
\end{gathered}
$$

Eliminating $a_{0}$ between (2) and (4) yields

$$
\begin{aligned}
z e^{-i \lambda} & =a_{1} e^{-i \beta_{1}}+a_{2} e^{-i \beta_{2}}-a_{2} e^{-i \beta_{2}} \omega^{5} \\
& =a_{1} e^{-i \beta_{1}}+\omega a_{2} e^{-\imath \beta_{2}}
\end{aligned}
$$

and hence, by complex conjugation,

$$
\begin{aligned}
z e^{i \lambda} & =a_{1} e^{i \beta_{1}}+\omega^{-1} a_{2} e^{i \beta_{2}} \\
& =a_{1} y_{1} e^{i \beta_{1}}+a_{2} y_{2} e^{i \beta_{2}}
\end{aligned}
$$

as required.

Now assume that the theorem is already proved for a value of $m-1$. Consider the configuration for $m$, as hitherto described. As noted, there is a positive integer $r, 1<r<m$, for which $P_{r}$ and $P_{r+1}$ are both joined to the same Steiner point $T$. Construct an equilateral triangle, with $P_{r} P_{r+1}$ as its base, with its other vertex $R$ so that $T$ and $R$ are on opposite sides of $P_{r} P_{r+1}$. Put

$$
\begin{aligned}
& a_{r-1}^{\prime}=\left|P_{r-1} R\right|, \\
& a_{r+1}^{\prime}=\left|R P_{r+2}\right|,
\end{aligned}
$$

and let $\beta_{r-1}^{\prime}, \beta_{r+1}^{\prime}$ be respectively the angles given by the directions of the vectors $\overrightarrow{P_{r-1} R}$ and $\overrightarrow{R P_{r+2}}$. Notice that $T$ is joined to just one other Steiner point $T_{1}$ (see Fig. 4).

As noted before, $R$ lies on the line $T_{1} T$ produced, such that

$$
\left|T P_{r}\right|+\left|T P_{r+1}\right|=|T R| \text {. }
$$




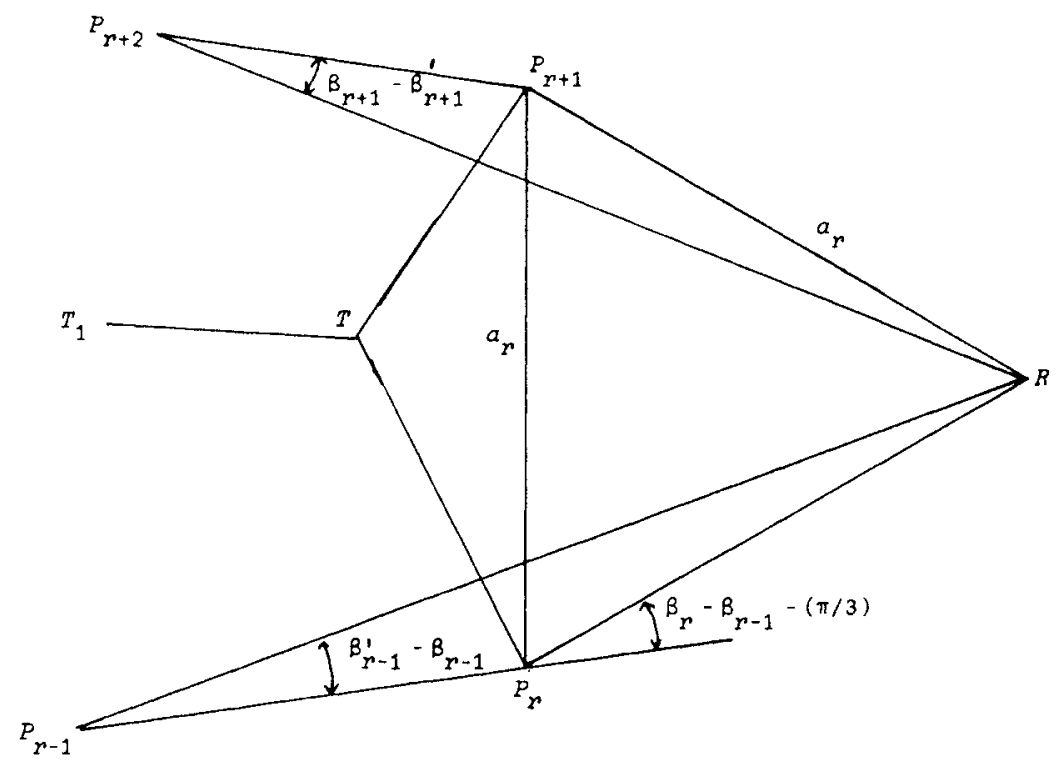

Fig. 4

The configuration, in which $P_{r-1} P_{r}, P_{r} P_{r+1}, P_{r+1} P_{r+2}$, is replaced by $P_{r-1} R, R P_{r+2}$, has one less segment and one less vertex than that given. Hence by the induction assumption,

$$
z e^{i \lambda}=\sum_{k=1}^{m-1} b_{k} z_{k} e^{i \gamma_{k}},
$$

where, from the construction,

$$
\begin{aligned}
& b_{k}=a_{k} \quad \text { if } \quad 1 \leq k \leq r-2, \\
& b_{r-1}=a_{r-1}^{\prime} \text {, } \\
& b_{r}=a_{r+1}^{\prime} \text {, } \\
& b_{k}=a_{k+1} \quad \text { if } \quad r+1 \leq k \leq m-1 \text {, } \\
& z_{k}=y_{k} \quad \text { if } \quad 1 \leq k \leq r-1 \text {, } \\
& z_{k}=y_{k+1} \quad \text { if } \quad r \leq k \leq m-1 \text {, }
\end{aligned}
$$

and

$$
\begin{aligned}
\gamma_{k} & =\beta_{k} \quad \text { if } \quad 1 \leq k \leq r-2, \\
\gamma_{r-1} & =\beta_{r-1}^{\prime}, \\
\gamma_{r} & =\beta_{r+1}^{\prime}, \\
\gamma_{k} & =\beta_{k+1} \quad \text { if } \quad r+1 \leq k \leq m-1 .
\end{aligned}
$$


Thus

$$
\begin{aligned}
z e^{i \lambda}= & \sum_{k=1}^{r-2} b_{k} z_{k} e^{i \gamma_{k}}+b_{r-1} z_{r-1} e^{i \gamma_{r-1}}+b_{r} z_{r} e^{i \gamma_{r}}+\sum_{k=r+1}^{m-1} b_{k} z_{k} e^{i \gamma_{k}} \\
= & \sum_{k=1}^{r-2} a_{k} y_{k} e^{i \beta_{k}}+a_{r-1}^{\prime} y_{r-1} e^{i \beta_{r-1}^{\prime}}+a_{r+1}^{\prime} y_{r+1} e^{i \beta_{r+1}^{\prime}}+\sum_{k=r+1}^{m-1} a_{k+1} y_{k+1} e^{i \beta_{k+1}} \\
= & \sum_{k=1}^{r-2} a_{k} y_{k} e^{i \beta_{k}}+y_{r-1}\left[a_{r-1} e^{i \beta_{r-1}}+a_{r} e^{i\left(\beta_{r}-\pi / 3\right)}\right] \\
& +y_{r+1}\left[a_{r} e^{i\left(\beta_{r}+\pi / 3\right)}+a_{r+1} e^{i \beta_{r+1}}\right]+\sum_{j=r+2}^{m} a_{j} y_{j} e^{i \beta,} \\
= & \sum_{k=1}^{r-1} a_{k} y_{k} e^{i \beta_{k}}+a_{r}\left[y_{r-1} \omega^{-1}+y_{r+1} \omega\right] e^{i \beta_{r}}+\sum_{k=r+1}^{m} a_{k} y_{k} e^{i \beta_{k}} \\
= & \sum_{k=1}^{m} a_{k} y_{k} e^{i \beta_{k}}
\end{aligned}
$$

by (1). This completes the proof.

Remark. If both $z$ and $\lambda$ are required, then the initial values $y_{1}=1, y_{m}=\omega^{-1}$ are both necessary. If $z$ alone is required, then $y_{1}=e^{i \alpha}, y_{m}=\omega^{-1} e^{i \alpha}$ for any real $\alpha$ will suffice.

Consider any spanning tree consisting of $m$ line segments joining the $(m+1)$ points $P_{0}, P_{1}, P_{2}, \ldots, P_{m}$. Each segment of the spanning tree can be described by a vector $d_{j} e^{i \delta}$, where $d_{j}$ is its length and $\delta_{j}$ determines its direction and sense.

Each vector $\overrightarrow{P_{k} P_{k+1}}$ is a unique linear combination of some vectors from the spanning tree, with coefficients \pm 1 . So, for each $k$,

$$
a_{k} e^{i \beta_{h}}=\sum_{j=1}^{m} \varepsilon_{k j} d_{j} e^{i \delta_{i}}
$$

where the coefficients $\varepsilon_{k j}=0,1$, or -1 are uniquely determined. This leads immediately to

Theorem 2. Let $d_{j} e^{i \delta}, j=1, \ldots, m$, describe the vectors of any spanning tree of the $(m+1)$ points $P_{0}, P_{1}, \ldots, P_{m}$. Then there exist scalars $x_{j}$, depending only on the configuration and the choice of spanning tree, such that

$$
z e^{i \lambda}=\sum_{j=1}^{m} d_{j} x_{j} e^{i \delta}
$$

Moreover, each $x_{j}$ belongs to the lattice $L$ defined by the three numbers 0,1 , and $\omega=e^{i \pi / 3}$. 
Proof. By Theorem 1 and equation (5),

$$
\begin{aligned}
z e^{i \lambda} & =\sum_{k=1}^{m} a_{k} y_{k} e^{i \beta_{k}} \\
& =\sum_{k=1}^{m} y_{k} \sum_{j=1}^{m} \varepsilon_{k j} d_{j} e^{i \delta_{j}} \\
& =\sum_{j=1}^{m} d_{j} e^{i \delta_{j}}\left[\sum_{k=1}^{m} y_{k} \varepsilon_{k j}\right] \\
& =\sum_{j=1}^{m} d_{j} x_{j} e^{i \delta_{j}}
\end{aligned}
$$

say, where $x_{j}=\sum_{k=1}^{m} y_{k} \varepsilon_{k j}$.

It is clear that $y_{1}=1 \in L, y_{m}=\omega^{-1} \in L$, and by the construction (1), each $y_{k} \in L$. Since $\varepsilon_{k j}=1,-1$ or 0 , each $x_{j} \in L$.

Example 1. Consider the full Steiner tree on four points $P_{0}, P_{1}, P_{2}, P_{3}$ as shown in Fig. 5. We have $y_{1}=1, y_{3}=\omega^{-1}$, so that

$$
\begin{aligned}
y_{2} & =\omega^{-1} y_{1}+\omega y_{3} \\
& =1+\omega^{-1} .
\end{aligned}
$$

By Theorem 1,

$$
z e^{i \lambda}=a_{1} e^{i \beta_{1}}+a_{2}\left(1+\omega^{-1}\right) e^{i \beta_{2}}+a_{3} \omega^{-1} e^{i \beta_{3}}
$$

Since

$$
a_{0}+a_{1} e^{i \beta_{1}}+a_{2} e^{i \beta_{2}}+a_{3} e^{i \beta_{3}}=0
$$

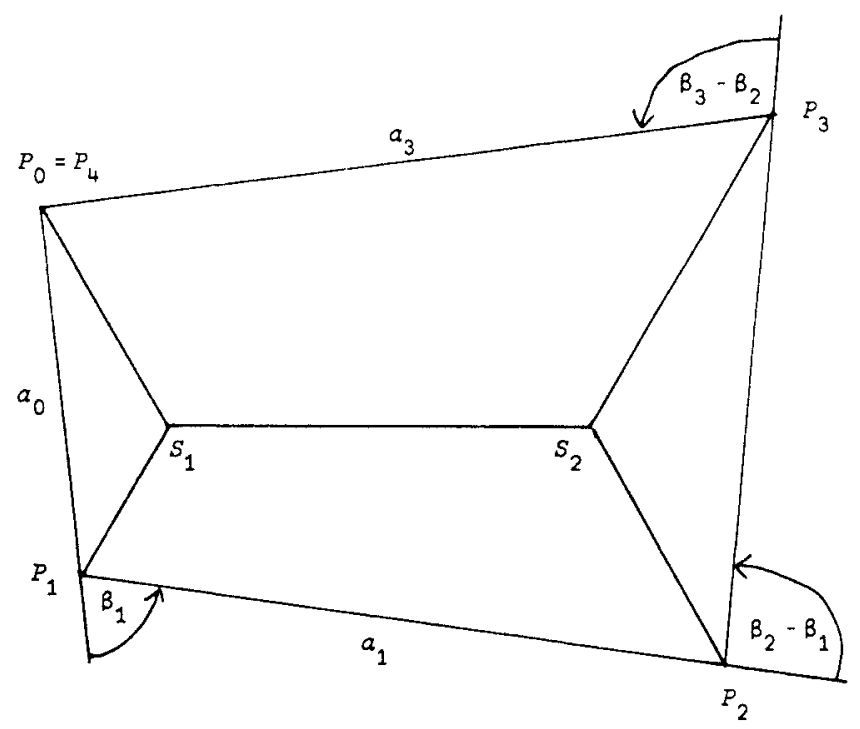

Fig. 5 
it also follows that

$$
\begin{aligned}
z e^{i \lambda} & =a_{1} e^{i \beta_{1}}+a_{2}\left(1+\omega^{-1}\right) e^{i \beta_{2}}+\omega^{-1}\left[-a_{0}-a_{1} e^{i \beta_{1}}-a_{2} e^{i \beta_{2}}\right] \\
& =\omega^{2} a_{0}+\omega a_{1} e^{i \beta_{1}}+a_{2} e^{i \beta_{2}}, \\
z \omega^{-2} e^{i \lambda} & =a_{0}+\omega^{-1} a_{1} e^{i \beta_{1}}+\omega^{-2} a_{2} e^{i \beta_{2}} .
\end{aligned}
$$

By taking complex conjugates and multiplying, we obtain

$$
\begin{aligned}
z^{2}= & a_{0}^{2}+a_{1}^{2}+a_{2}^{2}+2 a_{0} a_{1} \cos \left(60-\beta_{1}\right) \\
& +2 a_{1} a_{2} \cos \left(60+\beta_{1}-\beta_{2}\right)+2 a_{0} a_{2} \cos \left(120-\beta_{2}\right) \\
= & a_{0}^{2}+a_{1}^{2}+a_{2}^{2}+2 a_{0} a_{1} \cos \left(120-\alpha_{0}\right) \\
& +2 a_{1} a_{2} \cos \left(120-\alpha_{2}\right)+2 a_{0} a_{2} \cos \left(240-\alpha_{0}-\alpha_{2}\right),
\end{aligned}
$$

where $\alpha_{0}, \alpha_{2}$ are respectively the internal angles $\angle P_{0} P_{1} P_{2}$ and $\angle P_{1} P_{2} P_{3}$.

Example 2. In Example 1 let $\overrightarrow{P_{1} P_{3}}=a_{13} e^{1 \delta_{13}}$. Then

$$
\begin{aligned}
z e^{i A} & =\omega^{2} a_{0}+\omega a_{1} e^{i \beta_{1}}+a_{2} e^{i \beta_{2}} \\
& =\omega^{2} a_{0}+\omega a_{1} e^{i \beta_{1}}+\left[-a_{1} e^{i \beta_{1}}+a_{13} e^{i \delta_{13}}\right] \\
& =\omega^{2} a_{0}+\omega^{2} a_{1} e^{i \beta_{1}}+a_{13} e^{i \delta_{13}}
\end{aligned}
$$

or

$$
z \omega^{-2} e^{i \lambda}=a_{0}+a_{1} e^{i \beta_{1}}+\omega^{-2} a_{13} e^{i \delta_{13}}
$$

which determines $z$ in terms of the spanning tree $\overrightarrow{P_{0} P_{1}}, \overrightarrow{P_{1} P_{2}}$, and $\overrightarrow{P_{1} P_{3}}$.

By taking modulus, it follows that

$$
\begin{aligned}
z^{2}= & a_{0}^{2}+a_{1}^{2}+a_{13}^{2}+2 a_{0} a_{1} \cos \beta_{1} \\
& +2 a_{1} a_{13} \cos \left(\delta_{13}-\beta_{1}-120\right)+2 a_{0} a_{13} \cos \left(\delta_{13}-120\right) \\
= & a_{0}^{2}+a_{1}^{2}+a_{13}^{2}+2 a_{0} a_{13} \cos (60-\gamma) \\
& +2 a_{1} a_{13} \cos (120-\delta)+2 a_{0} a_{1} \cos (180-\delta-\gamma),
\end{aligned}
$$

where $\gamma=\angle P_{0} P_{1} P_{3}$ and $\delta=\angle P_{3} P_{1} P_{2}$.

Example 3. In Example 1 let $\overrightarrow{P_{2} P_{4}}=a_{24} e^{i \delta_{24}}$. Then, from Example 2,

$$
\begin{aligned}
z \omega^{-2} e^{i \lambda} & =-a_{24} e^{i \delta_{24}}+\omega^{-2} a_{13} e^{i \delta_{13},}, \\
z e^{i \lambda} & =a_{13} e^{i \delta_{13}}+\omega^{-1} a_{24} e^{i \delta_{24},},
\end{aligned}
$$

and hence

$$
z^{2}=a_{13}^{2}+a_{24}^{2}+2 a_{13} a_{24} \cos \left(\delta_{24}-\delta_{13}-60\right) .
$$


If the diagonals $P_{1} P_{3}$ and $P_{2} P_{4}$ meet at $I$, and $\angle P_{0} I P_{1}=\psi$, then this yields

$$
z^{2}=a_{13}^{2}+a_{24}^{2}+2 a_{13} a_{24} \cos (120-\psi)
$$

since $180-\psi=\delta_{24}-\delta_{13}$.

This formula shows that $z$ depends only on the lengths of the diagonals, and the angle between them. However, we should note that the two diagonals do not constitute a spanning tree. In general, we do not need to consider intersecting pairs of segments.

Corollary. It is well known [6] that for four given points, there are just two possible full Steiner trees, namely, the one just considered, as in Fig. 5, and the one illustrated in Fig. 6. By Example 3, with $\psi$ replaced by $180-\psi$, the length $z$ ' of this other Steiner tree is given by

$$
z^{\prime 2}=a_{13}^{2}+a_{24}^{2}+2 a_{13} a_{24} \cos (\psi-60)
$$

Consequently, $z \leq z^{\prime}$ if and only if $\cos (120-\psi) \leq \cos (\psi-60)$, that is, if and only if $\psi \leq 90^{\circ}$. In particular, the two Steiner trees have the same length when the diagonals meet at right angles.

Remark. This reproves Lemma 4 of Pollak's paper [9]. The results of Example 3 , and its corollary, including equation (7), also appear in Ollerenshaw's paper [8].

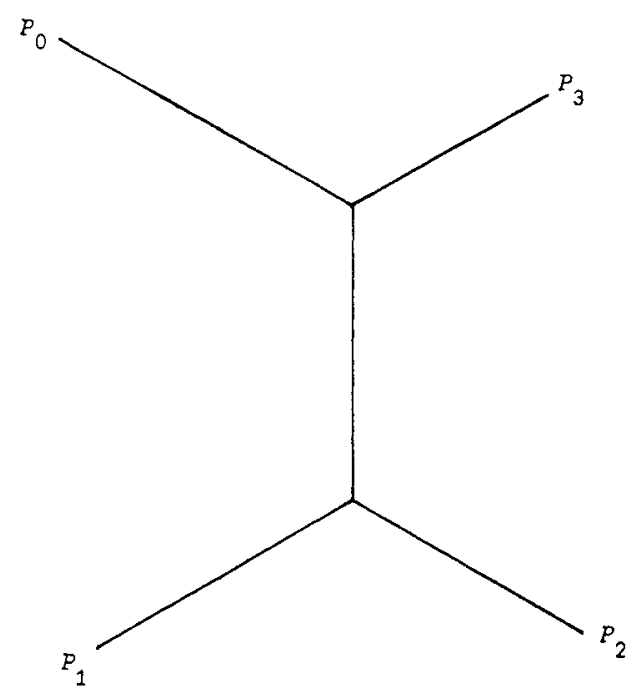

Fig. 6 


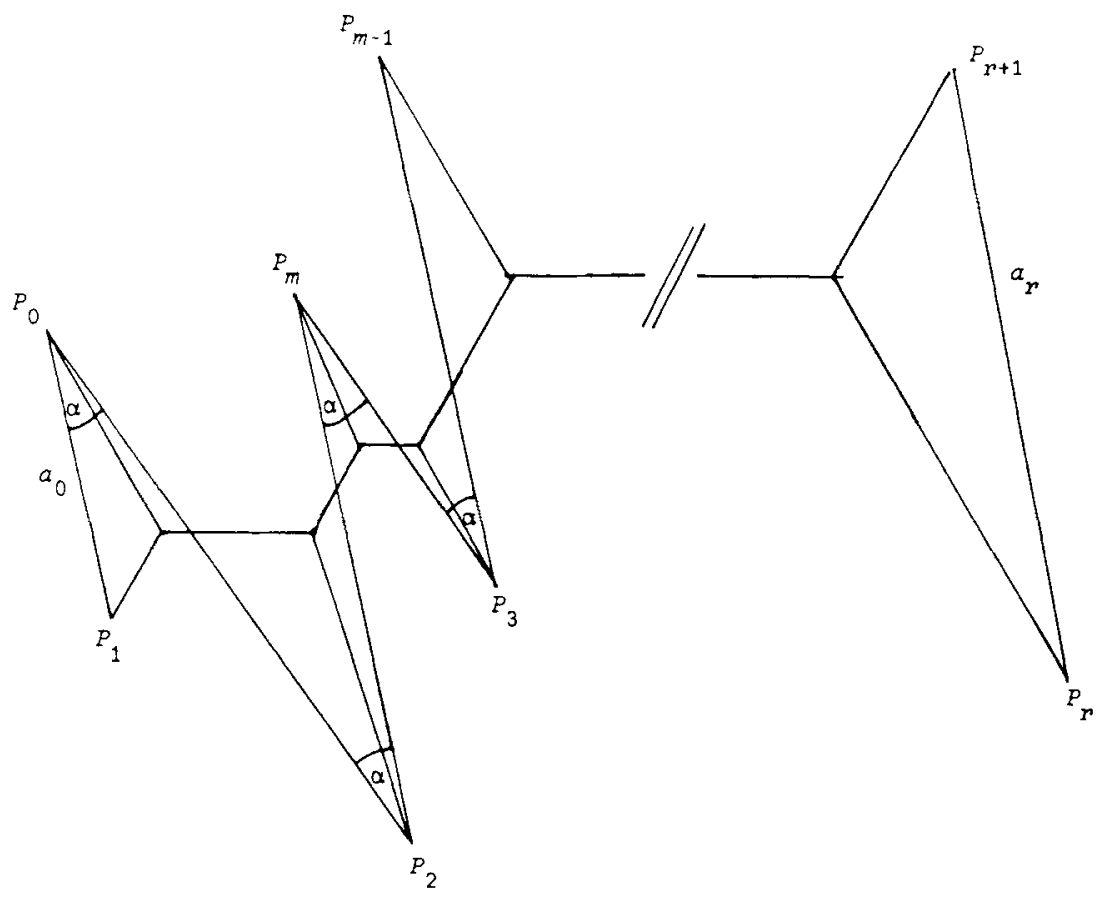

Fig. 7

Example 4. We consider the case where the Steiner points all lie on one path, with the path turning through angles $60^{\circ}$ anticlockwise at $S_{2}, S_{4}, S_{6}, \ldots$, and through $60^{\circ}$ clockwise at $S_{3}, S_{5}, \ldots$, as shown in Fig. 7. We assume that the number of Steiner points $m-1$ is even, and that $r=(m+1) / 2$.

The topology guarantees that $y_{1}=1, y_{m}=\omega^{-1}$ and

$$
\begin{gathered}
w^{-1} y_{1}+w y_{m}=1+w^{-1}=y_{2}, \\
w^{-1} y_{2}+w y_{m}=2 w^{-1}=y_{m-1}, \\
w^{-1} y_{2}+w y_{m-1}=1+2 w^{-1}=y_{3},
\end{gathered}
$$

and in general

$$
\begin{aligned}
y_{k} & =1+(k-1) w^{-1}, \quad k=1,2, \ldots, r, \\
y_{m-k+1} & =k w^{-1}, \quad k=1,2, \ldots,(r-1) .
\end{aligned}
$$

Now suppose further that the path $P_{1} P_{0} P_{2} P_{m} P_{3} P_{m-1} \cdots P_{r} P_{r+1}$ is a zigzag path with constant angle $\alpha$, at each $P_{k}$. Put

$$
\begin{array}{ll}
u_{k}=\left|P_{k} P_{m+2-k}\right|, & k=1,2, \ldots, r, \\
v_{k}=\left|P_{k} P_{m+3-k}\right|, & k=2, \ldots, r .
\end{array}
$$


By some elementary trigonometry,

$$
\begin{aligned}
a_{k} e^{i \beta_{k}} & =-u_{k}+v_{k+1} e^{i k x}, & k & =1,2, \ldots,(r-1), \\
a_{m-k+1} e^{i \beta_{m-k+1}} & =u_{k+1}-v_{k+1} e^{i \alpha}, & k & =1,2, \ldots,(r-1) .
\end{aligned}
$$

and

$$
a_{r} e^{i \beta_{r}}=-u_{r}
$$

It follows, by substitution, that

$$
\begin{aligned}
z e^{i \lambda}= & \sum_{k=1}^{m} a_{k} y_{k} e^{i \beta_{k}} \\
= & \sum_{k=1}^{r-1}\left(-u_{k}+v_{k+1} e^{i \alpha}\right)\left[1+(k-1) w^{-1}\right]-u_{r}\left[1+(r-1) w^{-1}\right] \\
& +\sum_{k=1}^{r-1}\left(u_{k+1}-v_{k+1} e^{i \alpha}\right)\left(k w^{-1}\right) \\
= & -\sum_{k=1}^{r} u_{k}+e^{i \alpha} w \sum_{k=1}^{r-1} v_{k+1}
\end{aligned}
$$

on simplification, Consequently,

$$
z e^{-i \lambda}=-\sum_{k=1}^{r} u_{k}+e^{-i \alpha} w^{-1} \sum_{k=1}^{r-1} v_{k+1}
$$

and hence

$$
z^{2}=\left(\sum_{k=1}^{r} u_{k}\right)^{2}+\left(\sum_{k=1}^{r-1} v_{k+1}\right)^{2}-2 \cos (60+\alpha)\left(\sum_{k=1}^{r} u_{k}\right)\left(\sum_{k=1}^{r-1} v_{k+1}\right)
$$

An easy modification deals similarly with the case when $m$ is even. This result may be compared with that recorded by Du et al. [3].

\section{Steiner Ratio for Quadrilaterals}

By employing some of the previous formulas, we can now present a short proof of the Steiner ratio conjecture for four points. We need one lemma.

Lemma. Let $b_{1} e^{i \varepsilon_{1}}, b_{2} e^{i \varepsilon_{2}}, \ldots, b_{m} e^{i \varepsilon_{m}}$ be given complex numbers, with $b_{i}>0$, $j=1, \ldots, m$. If

$$
\max _{1 \leq j<k \leq m}\left|\varepsilon_{j}-\varepsilon_{k}\right| \leq \pi / 3,
$$

then

$$
\left|\sum_{j=1}^{m} b_{j} e^{i \varepsilon_{j}}\right| \geq \frac{\sqrt{3}}{2} \sum_{j=1}^{m} b_{j}
$$


Proof. By a rotation and some relabeling if necessary, we can assume that

$$
0=\varepsilon_{1} \leq \varepsilon_{2} \leq \varepsilon_{3} \cdots \leq \varepsilon_{m} \leq \pi / 3 \text {. }
$$

But then $b_{1} e^{\prime f}=c_{1}+d_{j} e^{1 \pi / 3}$ uniquely, where $c_{1} \geq 0$ and $d_{j} \geq 0$ for each $j$, and

$$
b_{j} \leq c_{j}+d_{j}
$$

If we write $C=\sum_{j=1}^{m} c_{j}$ and $D=\sum_{j=1}^{m} d_{j}$, it then suffices to show

$$
\left|C+D e^{i \pi / 3}\right| \geq \frac{\sqrt{3}}{2}(C+D)
$$

for $C$ and $D \geq 0$. But this is equivalent to $(C-D)^{2} \geq 0$ by squaring and rearranging.

Theorem 3. Let $L_{\mathrm{s}}$ and $L_{\mathrm{m}}$ denote respectively the lengths of the Steiner minimal tree and the minimal spanning tree on four points. Then

$$
L_{\mathrm{s}} \geq(\sqrt{3} / 2) L_{\mathrm{m}}
$$

Proof. It is enough to consider full Steiner trees [6]. It is enough, in the notation of this paper, to prove that there is a spanning tree of length at most $(2 / \sqrt{3}) z$, for any given Steiner configuration. By the previous lemma, it is enough to write $z$ in the form

$$
z=\sum_{1=1}^{3} b_{1} e^{1 \varepsilon}
$$

where $b_{1}, b_{2}, b_{3}$ are the lengths of the segments of a spanning tree, with

$$
\max _{i \neq k}\left|\varepsilon_{j}-\varepsilon_{k}\right| \leq \pi / 3
$$

Consider the configuration illustrated in Fig. 5. It is immediate that $\beta_{2} \geq 60$. By symmetry, we may assume that $\beta_{2} \leq 180$ (otherwise interchange $P_{1} P_{2}$ with $P_{0} P_{3}$ ). Next we may assume $\beta_{1} \leq \beta_{2} / 2$ (otherwise interchange $P_{0} P_{1}$ with $P_{2} P_{3}$ ).

By Theorem 1,

$$
\begin{aligned}
z e^{i \lambda} & =a_{0}+a_{1} \omega^{-1} e^{i \beta_{1}}+a_{2} \omega^{-2} e^{i \beta_{2}} \\
& =a_{0} e^{i 0}+a_{1} e^{i\left(\beta_{1}-60\right)}+a_{2} e^{i\left(\beta_{2}-120\right)}
\end{aligned}
$$

Since $\left|\beta_{2}-120\right| \leq 60$, and $\left|\beta_{1}-60\right| \leq 60$ by choice, and $-60 \leq\left(\beta_{2}-120\right)-\left(\beta_{1}-60\right)$, the proof is complete if $\beta_{2}-\beta_{1} \leq 120$, by the above lemma. Otherwise, $\beta_{2}-\beta_{1}>$ 120 , whence

$$
\beta_{1}<\beta_{2}-120 \leq 180-120=60 \text {. }
$$


If $\delta_{13}-\beta_{1}>60$, then

$$
\begin{aligned}
z e^{i \lambda} & =a_{0}+\omega^{-1} a_{1} e^{i \beta_{1}}+\omega^{-2}\left[-a_{1} e^{i \beta_{1}}+a_{13} e^{i \delta_{13}}\right] \\
& =a_{0}+a_{1} e^{1 \beta_{1}}+a_{13} \omega^{-2} e^{i \delta_{13}}
\end{aligned}
$$

may be used. If not, then $\delta_{13}-\beta_{1}<60$, so $\beta_{2}-\delta_{13}>60$ and $\beta_{2}>\beta_{1}+120 \geq 120$, so

$$
\begin{aligned}
z e^{i \lambda} & =a_{0}+\omega^{-1}\left[a_{13} e^{i \delta_{13}}-a_{2} e^{i \beta_{2}}\right]+a_{2} \omega^{-2} e^{i \beta_{2}} \\
& =a_{0}+\omega^{-1} a_{13} e^{i \delta_{13}}+a_{2} \omega^{-3} e^{i \beta_{2}}
\end{aligned}
$$

can be used instead. This completes the proof.

\section{Acknowledgments}

The author wishes to acknowledge a debt to H. Pollak, M. Kaykobad, $H$. Rubinstein, and D. Thomas for some stimulating discussions on this topic. Much of the preparatory work for this paper was done while the author was on leave at the Mathematics Institute, University of Warwick, England, during the summer of 1986 . He wishes to express his warm appreciation of the hospitality provided by the Institute and its staff. Finally, thanks are due to the referee for some eminently suitable suggestions of improvement.

\section{References}

1. F. R. K. Chung and R. L. Graham, A new bound for Euclidean Steiner minimal trees, Ann. N. Y. Acad. Sci. 440 (1985), 328-346.

2. D. Z. Du and F. K. Hwang, A new bound for the Steiner ratio, Trans. Amer. Math. Soc. 278 , No. 1 (1983), 137-148.

3. D. Z. Du, F. K. Hwang, and J. F. Weng, Steiner minimal trees on zigzag lines, Trans. Amer. Math. Soc. 278, No. 1 (1983), 149-156.

4. D. Z. Du, E. Y. Yao, and F. K. Hwang, A short proof of a result of Pollak on Steiner minimal trees, J. Combin. Theory Ser. A 32 (1982), 396-400.

5. D. Z. Du, E. Yao, and F. K. Hwang, The Steiner ratio conjecture is true for five points, J. Combin. Theory Ser. A 38 (1985), 230-240.

6. E. N. Gilbert and H. O. Pollak, Steiner minimal trees, SIAM J. Appl. Math. 16 (1968), 1-29.

7. Z. A. Melzak. On the problem of Steiner, Canad. Math. Bull. 4 (1961), 143-148.

8. K. Ollerenshaw, Minimum networks linking four points in a plane, Bull. Inst. Math. Appl. 15, Nos. 8-9 (1979), 208-211.

9. H. O. Pollak. Some remarks on the Steiner problem, J. Combin. Theory Ser. A 24 (1978), 278-295.

Received July 15, 1988, and in revised form December 19, 1988. 